\title{
A Fuzzy Version of the TOPSIS Method in Multi- Criteria Decision-Making Tasks
}

\author{
Oleg Uzhga-Rebrov ${ }^{1}$, Galina Kuleshova ${ }^{2 *}$ \\ ${ }^{1}$ Rezekne Academy of Technologies, Rezekne, Latvia \\ ${ }^{2}$ Riga Technical University, Riga, Latvia
}

\begin{abstract}
Choice and decision making are an integral part of the purposeful activities of people in all areas of public and private life. Tasks of multi-criteria decision making are characterised by the fact that alternative decisions are evaluated by a set of criteria and the concept of a decision and its outcome coincide. The defining concept in such problems is the concept of a set of Pareto optimal decisions (Pareto set). This set forms alternative decisions that are not comparable in terms of the set of evaluation criteria. The choice of the optimal decision in the Pareto set can be performed only on the basis of the subjective preferences of the decision maker. In recent decades, extensions of traditional methods of multi-criteria decision making to a fuzzy environment have been proposed. One of the well-known approaches to multicriteria decision making is the TOPSIS method. In the paper, a fuzzy version of this method is considered in situations where the values of evaluation criteria are set in the form of fuzzy numbers.
\end{abstract}

Keywords - Fuzzy criteria values, ideal negative decision, ideal positive decision, multi-criteria decision making.

\section{INTRODUCTION}

In a broad sense, decision-making problems can be divided into two large classes:

(1) Problems of decision making in conditions of certainty, when the outcomes of alternative decisions are not influenced by any uncertain factors (events). In this kind of problems, the concepts of alternative decisions and their outcomes coincide.

(2) Problems of decision making under risk, when the outcomes of alternative decisions are caused by external random events (states of nature). Problems of the first class are usually called multi-criteria decision making, meaning that each decision is evaluated by a set of values of the relevant criteria [1]-[4].

The determining factor in multi-criteria decision making is the concept of a set of Pareto optimal alternative decisions (Pareto set). This set is formed of decisions that cannot be compared using only the values of the evaluation criteria. If we take as a basis any pair of decisions from the Pareto set, then one decision will be better than the other according to some criteria, but worse according to other criteria [5]-[8].

The choice of the optimal decision in the Pareto set can be carried out only on the basis of the subjective preferences of the decision maker. Whatever the system of subjective preferences is, it is always based on one of two global optimality principles:
(1) Achieving the maximum possible effect with a given cost. (2) Achieving the desired effect with the lowest possible cost. One of the well-known approaches to the multi-criteria choice of decisions is the TOPSIS method (Technique for Order Preference by Similarity to Ideal Decision). This method was first proposed in [9]. Further improvements of the method are presented in [9]-[11]. Among the latest works related to this method, [12], [13] must be mentioned. The essence of this method is as follows. On the basis of the existing criteria, assessments of alternative decisions, the so-called "ideal positive decision" and "ideal negative decision" are formed. All alternative decisions are evaluated on the basis of calculating the distances from these decisions to "ideal" decisions.

In real situations of making decisions, it is not always possible to set unambiguous criteria-based evaluations of decisions. This can be caused by various reasons: the undefined nature of the criteria themselves, the lack of initial data, the variability of the values of the criteria over time, and others. In such situations, it seems appropriate to model the initial uncertainty of the values of the criteria. One of the options for such modelling is to represent the values of the criteria in a fuzzy form. In recent decades, various extensions of traditional methods of multi-criteria decision analysis to a fuzzy environment have been proposed [14], [15].

In this paper, a fuzzy version of the TOPSIS method is considered; it is assumed that the values of the evaluation criteria are given in the form of triangular fuzzy numbers.

The objective of the paper is to present in detail theoretical foundations of the fuzzy version of the TOPSIS method and provide examples of its practical use for decision making in various areas of social activity.

Let us shortly outline the minimum necessary information about fuzzy numbers and operations on them, which will be used in the presentation of further material.

In general, a fuzzy number is a fuzzy set defined on the set of real numbers $\mathbf{R}$. A fuzzy number can be specified in the form of a set of values on $\mathbf{R}$ and the corresponding values of the membership function, in the form of a membership function graph, and in the form of an expression describing the membership function. As an example, Fig. 1 shows the graphs of the membership functions of two triangular fuzzy numbers $\tilde{A}$ and $\tilde{B}$.

*Corresponding author. E-mail: galina.kulesova@rtu.lv 


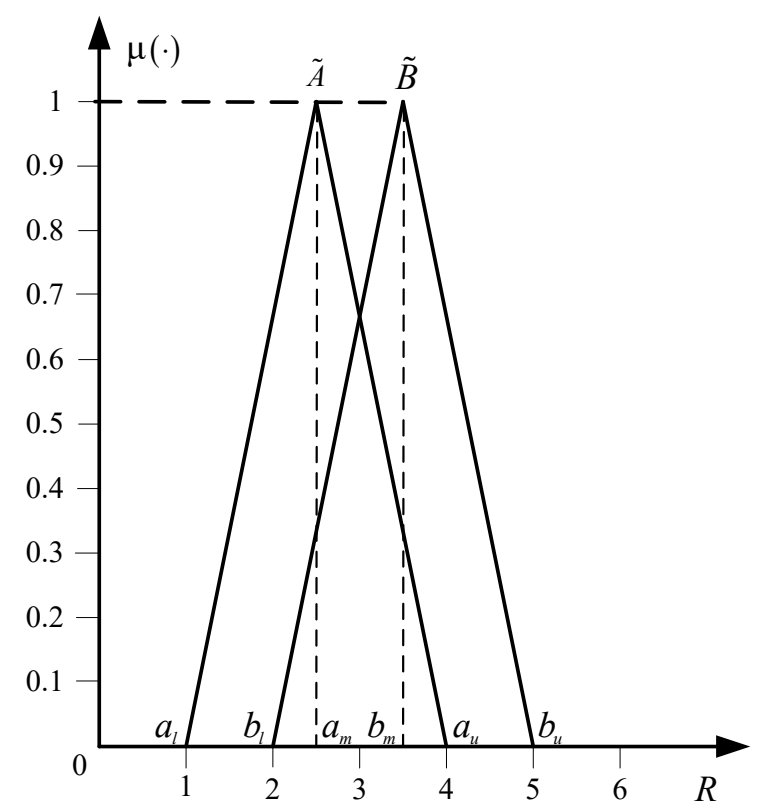

Fig. 1. Graphical representation of membership functions of two triangular fuzzy numbers $\tilde{A}$ and $\widetilde{B}$.

In Fig. 1, these denotations are used:

$a_{1}, b_{1}$ - lower bounds of supports of fuzzy numbers $\tilde{A}$ and $\tilde{B}$; $a_{m}, b_{m}-$ cores (modal values) of fuzzy values $\tilde{A}$ and $\tilde{B}$;

$a_{u}, b_{u}$-upper bounds of supports of fuzzy numbers $\tilde{A}$ and $\tilde{B}$.

Fuzzy numbers $\tilde{A}$ and $\tilde{B}$ that are graphically represented in Fig. 1 can be written in parametric form as $\tilde{A}=(1,2.5,4)$ and $\widetilde{B}=(2,3.5,5)$.

Let us define arithmetic operations on fuzzy numbers. Let two fuzzy numbers $\tilde{A}=\left(a_{l}, a_{m}, a_{u}\right)$ and $\tilde{B}=\left(b_{l}, b_{m}, b_{u}\right)$ be given. Then

$$
\begin{gathered}
-\tilde{A}=\left(-a_{u},-a_{m},-a_{l}\right) ; \\
\tilde{A}+\tilde{B}=\left(a_{l}+b_{l}, a_{m}+b_{m}, a_{u}+b_{u}\right) ; \\
\tilde{A}-\tilde{B}=\tilde{A}+(-\tilde{B})=\left(a_{l}-b_{u}, a_{m}-b_{m}, a_{u}-b_{l}\right) .
\end{gathered}
$$

In this paper, we use simplified versions of multiplication and division of fuzzy numbers. In the overwhelming majority of practical cases, these operations are correct, with the exception of some specific situations that are not typical in the context of problems of fuzzy choice of decisions.

$$
\begin{gathered}
\tilde{A} * \tilde{B}=\left(a_{l} * b_{l}, a_{m} * b_{m}, a_{u} * b_{u}\right), \tilde{A}>0, \tilde{B}>0 \\
\tilde{A} * \tilde{B}=\left(-a_{l} * b_{u},-a_{m} * b_{m},-a_{u} * b_{l}\right), \\
\tilde{A}>0, \tilde{B}<0, \text { or } \tilde{A}<0, \tilde{B}>0 . \\
\frac{\tilde{A}}{\tilde{B}}=\tilde{A} * \frac{1}{\tilde{B}}=\left(\frac{a_{l}}{b_{u}}, \frac{a_{m}}{b_{m}}, \frac{a_{u}}{b_{l}}\right) .
\end{gathered}
$$

\section{FUZZY VERSION OF TOPSIS}

Let the Pareto set containing $m$ alternative decisions be defined. These decisions are evaluated by a set $n$ of fuzzy criteria $\left\{\tilde{k}_{i j} / i=1, \ldots, m, j=1, \ldots, n\right\}$. To select the optimal decision using this approach, it is necessary to perform the following calculation procedures.

1. To calculate fuzzy normalised values of criteria $\tilde{r}_{j i}$ using Eq. (6)

$$
\tilde{r}_{j i}=\frac{\tilde{k}_{j i}}{\sqrt{\sum_{j=1}^{m} \tilde{k}_{j i}^{2}}}, i=1, \ldots, n .
$$

2. To calculate fuzzy weighted values of criteria using Eq. (7)

$$
\tilde{\mathrm{v}}_{j i}=\tilde{\alpha}_{i} \tilde{r}_{j i}, j=1, \ldots, m, i=1, \ldots, n \text {. }
$$

When the values of coefficients $\alpha_{i}$ are set in a deterministic form, the fuzzy weighted values of criteria are calculated using Eq. (8)

$$
\tilde{\mathrm{v}}_{j i}=\alpha_{i} \tilde{r}_{j i}, j=1, \ldots, m, i=1, \ldots, n
$$

3. To generate an ideal positive decision $\mathrm{a}^{+}$by condition (9)

$$
a^{+} \rightarrow\left\{\left(\max _{j} \tilde{v}_{j i} / i \in I\right),\left(\min _{j} \tilde{v}_{j i} / i \in I^{\prime}\right)\right\}, j=1, \ldots, m,(9)
$$

where $I$ - a subset of criteria representing positive effects; $I^{\prime}-$ a subset of cost criteria.

4. To generate an ideal negative decision $\mathrm{a}^{-}$by condition (10) 


$$
a^{-} \rightarrow\left\{\left(\min _{j} \tilde{v}_{j i} / i \in I\right),\left(\max _{j} \tilde{v}_{j i} / i \in I^{\prime}\right)\right\}, j=1, \ldots, m
$$

5. To calculate the distances between alternative decisions and ideal decisions (separation estimates). In general case, these distances can be calculated using the following expressions:

$$
\begin{aligned}
& S_{j}^{+}=\sum_{i=1}^{n} d\left(\tilde{\mathrm{v}}_{j i}, \tilde{\mathrm{v}}_{i}^{+}\right), j=1, \ldots, m ; \\
& S_{j}^{-}=\sum_{i=1}^{n} d\left(\tilde{\mathrm{v}}_{j i}, \tilde{\mathrm{v}}_{i}^{-}\right), j=1, \ldots, m,
\end{aligned}
$$

where: $d\left(\tilde{\mathrm{v}}_{j i}, \tilde{\mathrm{v}}_{i}^{+}\right)$- an estimate of the distance between the fuzzy weighted value of the $i$-th criterion of the $j$-th alternative decision and the fuzzy weighted value of the $i$-th criterion of the ideal positive decision $\mathrm{a}^{+} ; d\left(\tilde{\mathrm{v}}_{j i}, \tilde{\mathrm{v}}_{i}^{-}\right)$- an estimate of the distance between the fuzzy weighted value of the $i$-th criterion of the $j$-th alternative decision and the fuzzy weighted value of the $i$-th criterion of the ideal negative decision $\mathrm{a}^{-}$.

How can the distances (11a) and (11b) be calculated in practice? The use of standard estimates of the distances between fuzzy numbers seems to be inappropriate, since these estimates require knowledge of the values of the membership functions at given points. To get around this problem, alternative approaches for calculating the required distances are proposed. In this paper, we use the approach proposed in [16]. According to this approach, the distance between triangular fuzzy numbers $\tilde{A}$ and $\tilde{B}$ is calculated by Eq. (12)

$$
d(\tilde{A}, \tilde{B})=\sqrt{\frac{1}{3}\left(\left(a_{l}-b_{l}\right)^{2}+\left(a_{m}-b_{m}\right)^{2}+\left(a_{u}-b_{u}\right)^{2}\right)} .
$$

Using that estimate of the distance, separation estimates $S_{j}^{+}$ and $S_{j}^{-}$can be calculated by Eq. (13a) and (13b)

$$
\begin{array}{r}
S_{j}^{+}=\sum_{i=1}^{n} d\left(\tilde{\mathrm{v}}_{j i}, \tilde{\mathrm{v}}_{i}^{+}\right)= \\
\sum_{i=1}^{n} \sqrt{\frac{1}{3}\left(\left(\mathrm{v}_{j i l}-\mathrm{v}_{i l}^{+}\right)^{2}+\left(\mathrm{v}_{j i m}-\mathrm{v}_{i m}^{+}\right)^{2}+\left(\mathrm{v}_{j i u}-\mathrm{v}_{i u}^{+}\right)^{2}\right)} ; \\
S_{j}^{-}=\sum_{i=1}^{n} d\left(\tilde{\mathrm{v}}_{j i}, \tilde{\mathrm{v}}_{i}^{-}\right)= \\
\sum_{i=1}^{n} \sqrt{\frac{1}{3}\left(\left(\mathrm{v}_{j i l}-\mathrm{v}_{i l}^{-}\right)^{2}+\left(\mathrm{v}_{j i m}-\mathrm{v}_{i m}^{-}\right)^{2}+\left(\mathrm{v}_{j i u}-\mathrm{v}_{i u}^{-}\right)^{2}\right)} .
\end{array}
$$

Note that estimates $S_{j}^{+}$and $S_{j}^{-}$calculated by expressions $(13 \mathrm{a}, 13 \mathrm{~b})$ are ordinary real numbers, which facilitates further analysis and choice of the optimal decision.

6 . To calculate estimates of the relative proximity of each of the alternative decisions to the ideal positive decision

$$
C_{j}^{*}=\frac{S_{j}^{-}}{S_{j}^{+}+S_{j}^{-}}, j=1, \ldots, m,
$$

where values $S_{j}^{+}$and $S_{j}^{-}$are calculated by Eqs. (13a) and (13b), respectively.

The optimal decision is determined by the condition

$$
\operatorname{opt}\left(\alpha_{j}\right) \rightarrow \max _{j} C_{j}^{*}
$$

\section{ILLUSTRATIVE EXAMPLE}

The Pareto set is formed by three alternative decisions, $\alpha_{1}, \alpha_{2}$ and $\alpha_{3}$. These decisions are evaluated by the values of five fuzzy criteria $\tilde{k}_{1}, \ldots, \tilde{k}_{5}$. The fuzzy values of the criteria are given in Table I. The following deterministic values of the relative importance of the criteria are given: $\alpha_{1}=0.2, \alpha_{2}=0.1, \alpha_{3}=$ $0.3, \alpha_{4}=0.1$ and $\alpha_{5}=0.3$. It is necessary to determine the optimal decision based on the above fuzzy version of the TOPSIS method.

TABLE I

FuZZY VALUES OF CRITERIA FOR DECISIONS IN THE PARETO SET

\begin{tabular}{|c|c|c|c|c|c|}
\hline \multirow{2}{*}{ Decisions } & \multicolumn{5}{|c|}{ Criteria } \\
\hline & $\tilde{k}_{j 1}$ & $\tilde{k}_{j 2}$ & $\tilde{k}_{j 3}$ & $\tilde{k}_{j 4}$ & $\tilde{k}_{j 5}$ \\
\hline$a_{1}$ & $(9,10,11)$ & $(4,5,6)$ & $(-6,-5,-4)$ & $(13,14,15)$ & $(-9,-8,-7)$ \\
\hline$a_{2}$ & $(7,8,9)$ & $(5,6,7)$ & $(-5,-4,-3)$ & $(1213,14)$ & $(-11,-10,-9)$ \\
\hline$a_{3}$ & $(11,12,13)$ & $(6,7,8)$ & $(-7,-6,-5)$ & $(10,11,12)$ & $(-8,-7,-6)$ \\
\hline
\end{tabular}

Using Eqs. (6), (8), let us calculate the values $\tilde{r}_{j 1}$ and $\tilde{v}_{j 1}$ for criterion $\tilde{k}_{j 1}$ over the entire set of alternative decisions

$$
\begin{aligned}
& \sqrt{\sum_{j} k_{j 1}^{2}}=\sqrt{(9,10,11)^{2}+(7,9,9)^{2}+(11,12,13)^{2}}= \\
& =\sqrt{(81,100,121)+(49,64,81)+(121,144,169)}= \\
& =\sqrt{(251,308,371)}=(15.843,17 \cdot 550,19 \cdot 261) .
\end{aligned}
$$

$$
\begin{aligned}
& \tilde{r}_{11}=\frac{(9,10,11)}{(15.843,17.550,19.261)}= \\
& =\left(\frac{9}{19.261}, \frac{10}{17.550}, \frac{11}{15.843}\right)=(0.467,0.570,0.694) ; \\
& \tilde{r}_{21}=\frac{(7,8,9)}{(15.843,17.550,19.261)}= \\
& =\left(\frac{7}{19.261}, \frac{8}{17.550}, \frac{9}{15.843}\right)=(0.363,0.456,0.568)
\end{aligned}
$$




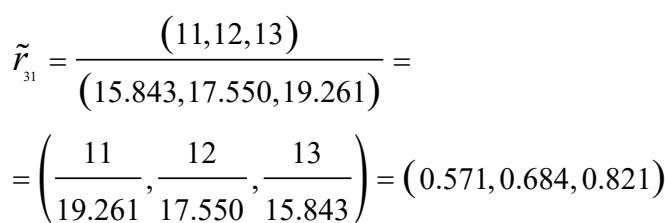

$$
\begin{gathered}
\tilde{\mathrm{v}}_{11}=\alpha_{1} \tilde{r}_{11}=0.2 \cdot(0.467,0.570,0.694)= \\
=(0.093,0.114,0.139) ; \\
\tilde{\mathrm{v}}_{21}=\alpha_{1} \tilde{r}_{31}=0.2 \cdot(0.363,0.456,0.568)= \\
=(0.073,0.091,0.114) ; \\
\tilde{\mathrm{v}}_{31}=\alpha_{1} \tilde{r}_{41}=0.2 \cdot(0.571,0.684,0.821)= \\
(0.114,0.137,0.164) .
\end{gathered}
$$

The calculated values are given in Table II.

TABLE II

CALCUlated VAlues For FuZzy VALUES OF CRITERION $\tilde{k}_{j 1}$ IN THE ILluStrative EXAMPLE

\begin{tabular}{|c|c|c|c|}
\hline Decisions & $\tilde{k}_{j 1}$ & $\tilde{r}_{j 1}$ & $\tilde{\mathrm{v}}_{j 1}$ \\
\hline$a_{1}$ & $(9,10,11)$ & $(0.467,0.570,0.694)$ & $(0.093,0.114,0.139)$ \\
\hline$a_{2}$ & $(7,8,9)$ & $(0.363,0.456,0.568)$ & $(0.073,0.091,0.114)$ \\
\hline$a_{3}$ & $(11,12,13)$ & $(0.571,0.684,0.821)$ & $(0.114,0.137,0.164)$ \\
\hline$\sqrt{\sum_{j}^{\tilde{k}_{j 1}^{2}}}$ & \multicolumn{3}{|c|}{$(15.843,17.550,19.261)$} \\
\hline$\tilde{\mathrm{v}}_{1}^{+}$ & \multicolumn{3}{|c|}{$(0.114,0.137,0.164)$} \\
\hline$\tilde{\mathrm{v}}_{1}^{-}$ & \multicolumn{3}{|c|}{$(0.073,0.091,0.114)$} \\
\hline
\end{tabular}

Additionally, the penultimate row of the table contains a fuzzy value, $\tilde{\mathrm{v}}_{1}^{+}$which is the maximum number, $\tilde{v}_{41}=\max _{j} \tilde{v}_{j i}$. The last line contains the fuzzy value $\tilde{v}_{1}^{-}$, which is the minimum value $\tilde{v}_{31}=\min \tilde{v}_{j i}$. These fuzzy values will be used later to form an ideal positive and ideal negative decision.

Calculations for other fuzzy criteria are performed in a similar way. The calculation results are given in Tables III-VI.

TABLE III

CALCUlated VAlues For Fuzzy VALUES OF CRITERION $\tilde{k}_{j 2}$

\begin{tabular}{|c|c|c|c|}
\hline Decisions & $\tilde{k}_{j 2}$ & $\tilde{r}_{j 2}$ & $\tilde{\mathrm{v}}_{j 2}$ \\
\hline$a_{1}$ & $(4,5,6)$ & $(0.328,0.477,0.684)$ & $(0.033,0.048,0.068)$ \\
\hline$a_{2}$ & $(5,6,7)$ & $(0.410,0.572,0,798)$ & $(0.041,0.057,0.080)$ \\
\hline$a_{3}$ & $(6,7,8)$ & $(0.491,0.67,0.912)$ & $(0.049,0.067,0.091)$ \\
\hline$\sqrt{\sum_{j} \tilde{k}_{j 2}^{2}}$ & & $(8.775,10.488,12.207)$ \\
\hline$\tilde{\mathrm{v}}_{2}^{+}$ & \multicolumn{3}{|c|}{$(0.049,0.067,0.091)$} \\
\hline$\tilde{\mathrm{v}}_{2}^{-}$ & \multicolumn{3}{|c|}{$(0.033,0.048,0.068)$} \\
\hline
\end{tabular}

TABLE IV

CAlculated VAlues For Fuzzy VALUES OF CRITERION $\tilde{k}_{j 3}$

\begin{tabular}{|c|c|c|c|}
\hline Decisions & $\tilde{k}_{j 3}$ & $\tilde{r}_{j 3}$ & $\tilde{\mathrm{v}}_{j 3}$ \\
\hline$a_{1}$ & $(-6,-5,-4)$ & $(-0.849,-0.570,-0.381)$ & $(-0.255,-0.171,-0.114)$ \\
\hline$a_{2}$ & $(-5,-4,-3)$ & $(-0.707,-0.456,-0.286)$ & $(-0.212,-0.137,-0.086)$ \\
\hline$a_{3}$ & $(-7,-6,-5)$ & $(-0.990,-0.684,-0.477)$ & $(-0.297,-0.205,-0.143)$ \\
\hline$\sqrt{\sum_{j} \tilde{k}_{j 3}^{2}}$ & \multicolumn{3}{|c|}{$(10.488,8.775,7.071)$} \\
\hline$\tilde{\mathrm{v}}_{3}^{+}$ & \multicolumn{3}{|c|}{$(-0.212,-0.137,-0.086)$} \\
\hline$\tilde{\mathrm{v}}_{3}^{-}$ & \multicolumn{3}{|c|}{$(-0.297,-0.205,-0.143)$} \\
\hline
\end{tabular}


TABLE V

CALCUlated VALUeS FOR FuZzy VALUES OF CRITERION $\tilde{k}_{j 4}$

\begin{tabular}{|c|c|c|c|}
\hline Decisions & $\tilde{k}_{j 4}$ & $\tilde{r}_{j 4}$ & $\tilde{\mathrm{v}}_{j 4}$ \\
\hline$a_{1}$ & $(13,14,15)$ & $(0.547,0.635,0.738)$ & $(0.055,0.063,0.074)$ \\
\hline$a_{2}$ & $(12,13,14)$ & $(0.505,0.590,0.689)$ & $(0.050,0.059,0.069)$ \\
\hline$a_{3}$ & $(10,11,12)$ & $(0.421,0.499,0.590)$ & $(0.042,0.050,0.059)$ \\
\hline$\sqrt{\sum_{j} \tilde{k}_{j 4}^{2}}$ & & $(20.322,22.045,23.770)$ \\
\hline$\tilde{\mathrm{v}}_{4}^{+}$ & & $(0.055,0.063,0.074)$ \\
\hline$\tilde{\mathrm{v}}_{4}^{-}$ & & $(0.042,0.050,0.059)$ \\
\hline
\end{tabular}

TABLE VI

Calculated Values for Fuzzy Values of CRiterion $\tilde{k}_{j 5}$

\begin{tabular}{|c|c|c|c|}
\hline Decisions & $\tilde{k}_{j 5}$ & $\tilde{r}_{j 5}$ & $\tilde{\mathrm{v}}_{j 5}$ \\
\hline$a_{1}$ & $(-9,-8,-7)$ & $(-0.699,-0.548,-0.429)$ & $(-0.210,-0.164,-0.129)$ \\
\hline$a_{2}$ & $(-11,-10,-9)$ & $(-0.854,-0.685,-0.552)$ & $(-0.256,-0.205,-0.166)$ \\
\hline$a_{3}$ & $(-8,-7,-6)$ & $(-0.621,-0.480,-0.368)$ & $(-0.186,-0.144,-0.110)$ \\
\hline$\sqrt{\sum_{j} \tilde{k}_{j 5}^{2}}$ & \multicolumn{3}{|c|}{$(16.310,14.595,12.884)$} \\
\hline$\tilde{\mathrm{v}}_{5}^{+}$ & \multicolumn{3}{|c|}{$(-0.186,-0.144,-0.110)$} \\
\hline$\tilde{\mathrm{v}}_{5}^{-}$ & \multicolumn{3}{|c|}{$(-0.256,-0.205,-0.166)$} \\
\hline
\end{tabular}

Using the data in Tables II-VI, let us form an ideal positive and ideal negative decision.

$$
\begin{aligned}
& a^{+} \rightarrow\left(\tilde{\mathrm{v}}_{1}^{+}, \tilde{\mathrm{v}}_{2}^{+}, \tilde{\mathrm{v}}_{3}^{+}, \tilde{\mathrm{v}}_{4}^{+}, \tilde{\mathrm{v}}_{5}^{+}\right)=((0.114,0.137,0.164), \\
& (0.049,0.067,0.091),(-0.212,-0.137,-0.086), \\
& (0.055,0.063,0.074),(-0.186,-0.144,-0.110)) \\
& a^{-} \rightarrow\left(\tilde{\mathrm{v}}_{1}^{-}, \tilde{\mathrm{v}}_{2}^{-}, \tilde{\mathrm{v}}_{3}^{-}, \tilde{\mathrm{v}}_{4}^{-}, \tilde{\mathrm{v}}_{5}^{-}\right)=((0.073,0.091,0.114), \\
& (0, .033,0.048,0.068),(-0.297,-0.205,-0.143), \\
& (0.042,0.050,0.059),(-0.256,-0.205,-0.166))
\end{aligned}
$$

Distances for other criteria are calculated in the same way. We have

$$
\begin{gathered}
d\left(\tilde{\mathrm{v}}_{12}, \tilde{\mathrm{v}}_{2}^{-}\right)=0, d\left(\tilde{\mathrm{v}}_{13}, \tilde{\mathrm{v}}_{3}^{-}\right)=0.03535 \\
d\left(\tilde{\mathrm{v}}_{41}, \tilde{\mathrm{v}}_{4}^{-}\right)=0.01339, d\left(\tilde{\mathrm{v}}_{15}, \tilde{\mathrm{v}}_{5}^{-}\right)=0.04151
\end{gathered}
$$

Using Eq. (12), we will calculate the distance from decision $a_{1}$ to the ideal positive decision $a^{+}$for each of the criteria.

$$
d\left(\tilde{\mathrm{v}}_{11}, \tilde{\mathrm{v}}_{1}^{-}\right)=\sqrt{\begin{array}{c}
1 / 3\left((0.093-0.073)^{2}+(0.114-0.091)^{2}+\right. \\
\left.(0.139-0.114)^{2}\right)=
\end{array}}
$$

$$
\begin{aligned}
& =\sqrt{\frac{1}{3}\left(0.020^{2}+0.023^{2}+0.025^{2}\right)}= \\
& =\sqrt{\frac{1}{3}(0.00040+0.00053+0.00062)}= \\
& =\sqrt{\frac{1}{3} \cdot 0.00155}=\sqrt{0.00052}=0.02280 .
\end{aligned}
$$

Distances for other criteria are calculated in the same way. We have

$$
\begin{array}{ll}
d\left(\tilde{\mathrm{v}}_{12}, \tilde{\mathrm{v}}_{2}^{-}\right)=0 ; & d\left(\tilde{\mathrm{v}}_{13}, \tilde{\mathrm{v}}_{3}^{-}\right)=0.03535 \\
d\left(\tilde{\mathrm{v}}_{41}, \tilde{\mathrm{v}}_{4}^{-}\right)=0.01339 ; & d\left(\tilde{\mathrm{v}}_{15}, \tilde{\mathrm{v}}_{5}^{-}\right)=0.04151 .
\end{array}
$$

For the remaining decisions, the distances to the ideal positive and ideal negative decisions are calculated in the same way. We have the following results.

- decision $a_{2}$ :

$$
\begin{gathered}
d\left(\tilde{\mathrm{v}}_{21}, \tilde{\mathrm{v}}_{1}^{+}\right)=0.04582 ; d\left(\tilde{\mathrm{v}}_{22}, \tilde{\mathrm{v}}_{2}^{+}\right)=0.00967 ; \\
d\left(\tilde{\mathrm{v}}_{23}, \tilde{\mathrm{v}}_{3}^{+}\right)=0 ; d\left(\tilde{\mathrm{v}}_{24}, \tilde{\mathrm{v}}_{4}^{+}\right)=0.00447 \\
d\left(\tilde{\mathrm{v}}_{25}, \tilde{\mathrm{v}}_{5}^{+}\right)=0.06261 .
\end{gathered}
$$

- decision $a_{3}$ :

$$
\begin{gathered}
d\left(\tilde{\mathrm{v}}_{31}, \tilde{\mathrm{v}}_{1}^{-}\right)=0 ; d\left(\tilde{\mathrm{v}}_{32}, \tilde{\mathrm{v}}_{2}^{-}\right)=0.00966 ; \\
d\left(\tilde{\mathrm{v}}_{33}, \tilde{\mathrm{v}}_{3}^{-}\right)=0.07092 ; d\left(\tilde{\mathrm{v}}_{34}, \tilde{\mathrm{v}}_{4}^{-}\right)=0.00894 ; \\
d\left(\tilde{\mathrm{v}}_{35}, \tilde{\mathrm{v}}_{5}^{-}\right)=0 .
\end{gathered}
$$


Using Eqs. (13a, 13b), let us calculate the values of the estimates of the separation $S_{1}^{+}, S_{1}^{-}$

$$
\begin{gathered}
S_{1}^{+}=\sum_{i=1}^{5} d\left(\tilde{\mathrm{v}}_{1 i}, \tilde{\mathrm{v}}_{i}^{+}\right)=0.03202+0.0194+0.03592+ \\
+0+0.02121=0.10864 . \\
S_{1}^{-}=\sum_{i=1}^{5} \begin{array}{c}
d\left(\tilde{\mathrm{v}}_{1 i}, \tilde{\mathrm{v}}_{i}^{-}\right)=0.02280+0+0.03535+ \\
+0.01379+0.04151=0.11345 .
\end{array}
\end{gathered}
$$

For other decisions, the values of the estimates of separation are calculated in the same way. We have

$$
\begin{gathered}
S_{2}^{+}=0.12257 ; S_{2}^{-}=0.08952 ; \\
S_{3}^{+}=0.08368, S_{3}^{-}=0.12802 .
\end{gathered}
$$

Using Eq. (14), let us calculate the estimate of the relative proximity of the decision $a_{1}$ to the ideal positive decision.

$C_{1}^{*}=\frac{S_{1}^{-}}{S_{1}^{+}+S_{1}^{-}}=\frac{0.11345}{0.10864+0.11345}=\frac{0.11345}{0.22209}=0.51083$.

By analogy, for decisions $a_{2}$ and $a_{3}$ we have $C_{2}^{*}=0.42208$, $C_{3}^{*}=0.60472$.

According to condition (15), $a_{3}$ should be chosen as the optimal decision.

\section{CONCLUSION}

Multi-criteria decision-making problems are specific problems in which each alternative decision is evaluated by a set of values of the relevant criteria and there are no uncertainties regarding the outcomes of these decisions. The determining factor in problems of this kind is the concept of the Pareto set, which is formed by decisions with contradictory values of the criteria. In other words, one alternative decision is better than the other according to some criteria, but worse on other criteria. The optimal decision can only be found in the Pareto set. We do not consider situations when in the original set of alternative decisions there is a globally optimal decision that is better than all other decisions for the entire set of criteria; these situations are the exception, not the rule.

The choice of the optimal decision in the Pareto set can only be carried out on the basis of the system of subjective preferences of the decision maker. All known formal approaches to multi-criteria decision making use specific methods for modelling subjective preferences.

The widely used formal methods for choosing decisions in the Pareto set are the additive and multiplicative convolution of criterion values, the method of compromise programming, the method of value function and the TOPSIS method.

A big problem in the tasks of multi-criteria choice of solutions is represented by the uncertainties regarding the values of the evaluation criteria. The use of fuzzy criteria values instead of the deterministic ones makes it possible to successfully model a wide range of initial uncertainties.
In this paper, a fuzzy version of the TOPSIS method has been considered. As in its non-fuzzy version, the comparison of alternative decisions in the Pareto set has been made on the basis of a combined estimate of the fuzzy distance from the estimated decision to the ideal positive and ideal negative decisions. Modelling the subjective preferences of the decision maker has been carried out using the coefficients of the relative importance of the criteria. Using distances to "ideal" decisions also simulates such subjective preferences.

Let us compare the fuzzy version of the TOPSIS method with fuzzy versions of additive and multiplicative convolution of criteria values. When using one or another type of convolution of criteria values, the resulting estimates have a fuzzy form, which makes it necessary to compare fuzzy numbers, which is a rather complex computational problem. The advantage of the TOPSIS method is that the final analysis of decisions and the choice of the optimal decision are based on deterministic resulting estimates, which greatly facilitates the implementation of these important procedures. The disadvantage of the TOPSIS method is a rather large amount of intermediate calculations required.

A fuzzy version of the TOPSIS method has found widespread use in decision making in various fields of human activity under conditions of uncertainty. Among the many works on this topic, mention should be made of the works [17][21].

\section{REFERENCES}

[1] E. Triantaphyllou, Multi-Criteria Decision Making Methods: A Comparative Study. USA: Springer Science+Business Media, 2000. https://doi.org/10.1007/978-1-4757-3157-6

[2] A. Tshizaka and Ph. Memery, Multi-Criteria Decision Analysis: Methods and Software. John Wiley \& Sons, Ltd., 2013. https://doi.org/10.1002/9781118644898

[3] N. Munier, E. Honotorio, and F. Jiménes-Sáez, Strategic Approach in Multi-Criteria Decision Making. A Practical Guide for Complex Scenarios. Switzerland: Springer, 2019. https://doi.org/10.1007/978-3030-02726-1

[4] O. Uzhga-Rebrov, Vibor Reshenij. Rezekne: RA Drukatava, 2016.

[5] S. Hiwa., T. Hiroyasu, and M. Miki, "Design mode analysis of Pareto solution set for decision-making support," Journal of Applied Mathematics, vol. 2014, Article ID 520209, Nov. 2014. https://doi.org/10.1155/2014/520209

[6] I. Giagkiozis and P. J. Fleming, "Pareto front estimation for decision making," Evol. Comput., vol. 22, no. 4, pp. 651-678, Dec. 2014. https://doi.org/10.1162/EVCO a 00128

[7] V. D. Noghin, Reduction of the Pareto Set. An Axiomatic Approach. Springer, 2018. https://doi.org/10.1007/978-3-319-67873-3

[8] M. Jankowski, A. Borsukievicz, and K. Hooman, "Development of decision-making tool and Pareto set analysis for di-objective optimization in ORS power plant," Energies, vol. 13, no. 20, p. 5280, Oct. 2020. https://doi.org/10.3390/en13205280

[9] C. L. Hwang and K. Yoon, Multiple Attribute Decision Making Methods and Applications. New York: Springer Verlag, 1981. https://doi.org/10.1007/978-3-642-48318-9

[10] K. Yoon, "A reconciliation among discrete compromise situations," Journal of Operational Research Society, vol.38, no. 3, pp. 277-281, Mar. 1987. https://doi.org/10.2307/2581948

[11] C. L. Hwang, Y. J. Lai, and T. Y. Liu, "A new approach for multiple objective decision making," Computers and Operational Research, vol. 20, no. 8, pp. 889-899, Oct. 1993. https://doi.org/10.1016/0305-0548(93)90109-V

[12] Z. Pavić and V. Novoselac, "Notes on TOPSIS method," Int. Journal of Engineering Research and General Science, vol. 1, no. 2, pp. 5-12, Jan. 2013. 
[13] Y. Çelikbilek and F. Tüysüz, "An in-depth review of theory of the TOPSIS method: An experimental analysis," Journal of Management Analytics, vol. 7, no. 2, pp. 281-300, Apr. 2020. https://doi.org/10.1080/23270012.2020.1748528

[14] G. Kahraman, Ed., Fuzzy Multi-Criteria Decision Making. New York: Springer-Verlag, Inc., 2010

[15] I. Kaya, M. Çolak, and F. Terzi, "A comprehensive review of fuzzy mult criteria decision making methodologies for energy policy making," Energy Strategy Reviews, vol. 24, pp. 207-228, Apr. 2019. https://doi.org/10.1016/j.esr.2019.03.003

[16] C. T. Chen, "Extension of the TOPSIS for group decision-making under fuzzy environment," Fuzzy Sets and Systems, vol. 114, no. 1, pp. 1-9, Aug. 2000. https://doi.org/10.1016/S0165-0114(97)00377-1

[17] Y.-M. Wang and N. M. S. Elhag, "Fuzzy TOPSIS method based on alpha level sets with an application to bridge risk assessment," Expert Systems with Applications, vol. 31, no. 2, pp. 309-319, Aug. 2006. https://doi.org/10.1016/j.eswa.2005.09.040

[18] T.-C. Chu and Y.-C. Lin, "A fuzzy TOPSIS method for robot selection," Int. Journal of Advanced Manufacturing Technology, vol. 21, pp. 284290, Feb. 2003. https://doi.org/10.1007/s001700300033

[19] Ü. Şengül, M. Eren, S. E. Shizar, V. Gezder, and A. Şengül, "Fuzzy TOPSIS method for ranking renewable energy supply systems in Turkey," Renewable Energy, vol. 75, pp. 617-625, Mar. 2015. https://doi.org/10.1016/i.renene.2014.10.045

[20] Ch.-CH. Lo, D.-Y. Chen, Ch.-F. Tsai, and K.-Ch. Chao, "Service selection based on fuzzy TOPSIS method," in Proceedings of the 2010 IEEE $24^{\text {th }}$ Conference on Advanced Information Networking and Applications Workshops, Perth, WA, Australia, Apr. 2010, pp. 367-372.

[21] A. Mayyas, M. A. Omar, and M. T. Hayajneh, "Eco-material selection using fuzzy TOPSIS method," Int. Journal of Sustainable Engineering, vol. 9, no. 5, pp. 292-304, Mar. 2016.

https://doi.org/10.1080/19397038.2016.1153168
Oleg Uzhga-Rebrov is a Leading Researcher of the Information and Communication Technologies Research Centre at Rezekne Academy of Technologies (Latvia). He received his Doctoral degree in Information Systems from Riga Technical University in 1994. His research interests include different approaches to processing incomplete, uncertain and fuzzy information, in particular, fuzzy set theory, rough set theory as well as fuzzy classification and fuzzy clustering techniques and their applications in bioinformatics. Currently he focuses on the problems of data analysis.

E-mail: rebrovs@tvnet.lv

Galina Kuleshova is a Researcher of the Faculty of Computer Science and Information Technology at Riga Technical University (Latvia). She received her M. Sc. degree in Decision Support Systems from Riga Technical University. Current research interests include artificial neural networks, data mining, ontology engineering, classification methods, data analysis and bioinformatics. E-mail: galina.kulesova@rtu.lv

ORCID iD: https://orcid.org/0000-0002-2048-9734 